\title{
La oportunidad perdida para la gobernanza participativa y la sostenibilidad en el ámbito local: la Agenda 21
}

\author{
The missed opportunity for sustainability \\ and governance participatory at the local level: \\ the Agenda 21 \\ Néstor García Montes \\ Universidad Complutense de Madrid, España \\ nestorga@ucm.es
}

\begin{abstract}
Resumen: La Agenda 21 ha sido una de las iniciativas para promover el desarrollo sostenible que mayor acogida e implementación ha tenido a nivel internacional desde que se puso en marcha a partir de la Cumbre de la Tierra de 1992. Y una de sus principales características ha sido el llamamiento a la participación de la comunidad local. Se trataba, por tanto, de un dispositivo con un gran potencial para la gobernanza y gestión participativa, pero que, en muchos casos, no ha sido plenamente aprovechado. A pesar de ciertos logros importantes, se podría decir que se ha quedado en una ocasión perdida. Algunas de las razones para que la participación haya sido, en gran medida, una asignatura pendiente en los procesos de la Agenda 21 en entornos locales, se analizan en el presente artículo.
\end{abstract}

Palabras clave: Participación, Agenda 21, sustentabilidad, local.

Abstract: Agenda 21 has been one of the initiatives to promote sustainable development and implementation greater acceptance internationally has had since it was launched from the Earth Summit 1992. And one of the main features of the Agenda 21 has been the call for participation of the local community. It was therefore a device with great potential for participatory governance and management but in many cases, has not been fully exploited. Despite some significant achievements, it has been a missed opportunity. Some reasons for participation have been largely a slope in the processes of Agenda 21 in local sites subject are discussed in this article.

Keywords: Participation, Agenda 21, sustainability, local. 


\section{SURGIMIENTO Y ENFOQUE DE LA AGENDA 21}

Las ciudades postindustriales se han constituido en el principal escenario de los efectos nocivos sobre el medio ambiente de la actividad humana, en el marco del modelo de crecimiento económico en el que se inscriben desde la Revolución Industrial. Las ciudades son los focos que más contribuyen directa e indirectamente al deterioro ecológico del planeta, consumiendo recursos naturales y generando deshechos y contaminación (Foladori, 2001). Por otro lado, la sociedad en su conjunto tiene un papel fundamental en la crisis ecológica actual, tanto en su estímulo y profundización como en su contención o inhibición. La iniciativa de la Agenda 21 nació con la pretensión de conjugar la sustentabilidad de los hábitats humanos con la implicación proambiental de la sociedad, responsable de la crisis ecológica. El principal papel en la cuestión ambiental lo tiene la sociedad en su conjunto, y aunque la Agenda 21 se orientó hacia un modelo de participación de la comunidad local en un sentido amplio (incluyendo a la ciudadanía de base, el tejido asociativo, los agentes económicos y sociales y las Administraciones públicas), en este caso se centrará el foco de análisis en las posibilidades y alcances de la participación de la base social (ciudadanía no organizada) y el tejido asociativo.

Conviene señalar que la Agenda 21 se puede definir como un plan de acción para el desarrollo sostenible (Jones y Stokes, 1998). Una herramienta de planificación y gestión ambiental orientada hacia la sustentabilidad desde el nivel local, espacio propicio para un desarrollo que establezca una interrelación local-global (Twyman, 1998); un desarrollo que promueva la satisfacción de las necesidades básicas de la población de manera justa y equitativa, sin degradar o agotar el capital natural que provee de recursos (Tyler Miller, 2007).

Ciertamente, el concepto de desarrollo sostenible ha suscitado numerosas críticas, aunque también es cierto que ha conseguido adherir prosélitos, especialmente entre los partidarios de la denominada sostenibilidad débil. El desarrollo sostenible defendía en su propósito el mantenimiento de un crecimiento económico, pero tratando de atenuar los efectos más devastadores sobre el medio ambiente. Desde diferentes planteamientos se ha cuestionado la pertinencia de vincular la idea de crecimiento económico con la sostenibilidad ambiental, y las principales críticas han llegado desde sectores y argumentos defensores de la denominada Sostenibilidad Fuerte. Mientras que la Débil contempla la compatibilidad de la sostenibilidad socioecológica con el modelo económico y de consumo capitalista, realizando los ajustes pertinentes para minimizar el impacto ambiental y en el uso de los recursos, la Sostenibilidad Fuerte aboga por un replanteamiento, incluso por una superación, del modelo de producción y crecimiento imperante desde la Revolución Industrial, por ejemplo fomentando actitudes y modos de vida de- 
crecentistas, o al menos de crecimiento 0 . Se podría decir que, mientras en la vertiente Débil de la sostenibilidad se antepone lo económico a lo ecológico, en la Fuerte ocurre lo contrario, considerando en este último caso que la solución de raíz de la crisis ecológica solo se puede lograr a partir de un cambio en el patrón de crecimiento. Algunas de las principales críticas que se han vertido sobre el concepto y el planteamiento del desarrollo sostenible se construyen en torno a la primacía del interés económico frente al ecológico, pero también se ha señalado que se trata de un concepto ambiguo, excesivamente abierto y poco comprometido, un cliché, un oxímoron y que es difícil de operativizar, medir y llevarlo a la práctica Sin embargo, a pesar de las diferentes críticas, se debe poner en la balanza positiva del concepto su incorporación a los programas de Naciones Unidas en el plano institucional (Mebratu, 1998). También su capacidad de incidir en los Gobiernos y agencias internacionales, como el Banco Mundial, para que pusieran atención en la preocupación ambiental (Arboit y De Rosa, 2014). Así se demostró poco después, en la Cumbre de la Tierra, donde la Agenda 21 se convirtió en una de las puntas de lanza para promover tal desarrollo sostenible.

La Agenda 21 es, quizás, una de las iniciativas que más repercusión ha tenido en la sustentabilidad local (Font, 2000) y surgió, bajo la denominación de Programa 21, en la Conferencia de Naciones Unidas sobre Medio Ambiente y Desarrollo (Cumbre de la Tierra) celebrada en Río de Janeiro en 1992. Integra las consideraciones y debates respecto a la necesidad de actuar desde lo local y con la implicación de toda la sociedad en los problemas de insustentabilidad ambiental que ya se venían fraguando desde la Cumbre celebrada veinte años antes en Estocolmo. La iniciativa fue aprobada por los representantes de los 178 países participantes en Río (Verdaguer, 2000), simbolizando un consenso internacional y un compromiso político al más alto nivel para la cooperación en los temas ambientales (Pellizzoni, 2001). Los acuerdos alcanzados se materializaron en cinco apartados, siendo el quinto el Programa 21, del que deriva la Agenda 21; un plan de acción de las Naciones Unidas para un desarrollo sostenible en el siglo xxI.

El planteamiento fundacional de la Agenda 21, además, requería de la implicación de la población local como rasgo característico (Bosworth, 1993), para lo que instaba a los poderes locales a iniciar un proceso de diálogo con la ciudadanía, organizaciones y entidades (O’Riordan y Voisey, 1998). «Sin participación social, sobre todo en la medida en que las acciones se concreten a nivel de estado, municipio o comunidad, donde los representantes de la comunidad debieran estar cada vez más cerca de sus representados, la gestión ambiental pierde sentido en cuanto formulación de políticas, estrategias y tácticas orientadas a la generación de procesos de desarrollo ambientalmente sustentable» (Robles de Benito et al., 2008: 51). Por tanto, la Agenda 21 se materializa en un plan medioambiental para utilizar los recursos de la manera más 
sustentable y eficiente posible, y conseguir la participación de la comunidad (Hewitt, 1995). En este sentido, el capítulo 28 del Programa 21 hace un llamamiento a todas las comunidades locales para que elaboren su propia Agenda 21 Local y traduzcan los objetivos generales y la filosofía del proyecto en planes y actuaciones locales y territorialmente aplicables. La importancia dada, al menos a priori y a nivel teórico, a la participación social en las Agendas 21 Locales queda reflejada en la dedicatoria de una de las cuatro secciones que componen el Programa 21 al fortalecimiento de los grupos principales. En ella se plasma la necesidad de que las personas, los grupos y las organizaciones participen en los procedimientos de evaluación del impacto ambiental, conozcan el mecanismo de adopción de decisiones y participen en él, sobre todo cuando exista la posibilidad de que esas decisiones afecten a las comunidades donde viven y trabajan (ONU, 1992).

La Agenda 21 Local propiciaba el escenario adecuado para hacer realidad los propósitos de promover una gestión participativa y una corresponsabilidad ciudadana (centrada en la sustentabilidad medioambiental), en sintonía con una democracia directa o democracia participativa que complemente a la democracia representativa (Habermas, 1998). Sin embargo, se considera que ese potencial no fue plenamente aprovechado y desplegado, quedando, en muchos casos, en una tentativa participativa más que en procesos de verdadera democracia directa que fueran más allá del modelo representativo y delegativo de las decisiones a que se refirió Schumpeter (1983 [1942]). Desde las premisas de la democracia representativa, la elección por sufragio de los representantes supone la principal decisión a la que debe someterse la ciudadanía (Dahl, 1987), y el funcionamiento democrático se garantiza en la medida en que todos los ciudadanos mayores de edad puedan ejercer ese derecho al voto. Por su parte, la democracia representativa no arroja todo el valor democrático en un único acto decisorio de carácter electoral. Plantea el enriquecimiento del sistema a través de la implicación y coparticipación de la ciudadanía en los asuntos públicos de una forma más intensa, regular y directa. «El dilema entre representación y participación suele resolverse a partir del argumento de la complementación» (Díaz Aldret, 2017: 344), es decir, en la compatibilización y conjugación de la representatividad con elementos y fórmulas deliberativos y participativos. Por tanto, ambos planteamientos (democracia representativa y participativa) no tienen por qué ser excluyentes, más bien al contrario, deben conciliarse. En esta conciliación, la Agenda 21 aportó su metodología y sus objetivos para, en el marco de un sistema representativo, abrir espacios participativos para la cocreación colectiva de políticas públicas, en este caso orientadas a la sostenibilidad local. Esta era una gran potencialidad de la Agenda 21; cuestión distinta es el aprovechamiento que se hiciera de la misma. 


\section{LA AGENDA 21: UNA INICIATIVA A NIVEL MUNDIAL}

Desde el momento de su nacimiento se produjo un espectacular crecimiento en cuanto al número de Agendas 21 Locales puestas en marcha en todo el mundo, alcanzando su mayor auge en la década de 2000. En esos años se convirtió en la «herramienta más generalizada e importante tanto de la planificación pública del desarrollo sostenible como de la participación ciudadana» (Garrido, 2005: 56). Esta efervescencia ponía de manifiesto el incremento de la preocupación social y política por la sustentabilidad en el ámbito local, donde, movidos por una demanda ciudadana creciente y una «moda» municipal, los partidos políticos y las autoridades locales incorporaron la Agenda 21 en sus programas y estrategias de gestión, constituyendo un elemento importante de las campañas electorales y del discurso de gobierno. Así, en todos los continentes se encuentran experiencias de Agenda 21, aunque Europa destaca especialmente, y, dentro de ésta, España ${ }^{1}$, uno de los países más fructíferos, junto con Italia y Francia (Font, 2000).

A pesar de que «resulte prácticamente imposible conocer con exactitud el número de Agendas 21 iniciadas» (Garrido, 2005: 56), en 1997, momento todavía de arranque incipiente de la iniciativa, la ONU, en su informe «Overall Assesment of progress achieved since UNCED», estimaba que unos dos mil gobiernos locales de 64 países de todo el mundo habían puesto en marcha sus procesos con cierta participación de la comunidad local. Algo más tarde, en 2001 (en la fase de despegue masivo del proyecto), el Consejo Internacional de Iniciativas Locales por el Medio Ambiente - ICLEI- contabilizaría 6.416 prácticas en 113 países diferentes (Bárcena, 2006), la inmensa mayoría localizadas en Europa (5.292). La segunda mitad de la década de 2000 fueron años de esplendor para la Agenda 21. En España, por ejemplo, creció exponencialmente el número de localidades que comenzaron a implementarla, al menos en una fase embrionaria $^{2}$. El Observatorio de la Sostenibilidad de España (OSE) llegó a contabilizar 3.763 en 2009, momento álgido del proyecto. Por su parte, en América Latina se pueden encontrar Agendas 21 en muchos países, como Bolivia, Brasil, Colombia, Perú, Chile, Nicaragua o Ecuador (Coria, 2007). En México se puede destacar la Agenda 21 para el Tu-

${ }^{1}$ El autor de este artículo ha participado como asesor metodológico y asistencia técnica en procesos de Agenda 21 en once ciudades de España: Aranjuez, Arganda del Rey, Fuenlabrada, Jaén, Madrid, Olmeda de las Fuentes, Pinto, Serranillos del Valle, Torrejón de Ardoz, Villalbilla y Villanueva del Pardillo.

${ }^{2}$ Como podía ser iniciar el Diagnóstico, la primera fase de la Agenda 21 con la que se pretendía conocer la realidad local para, posteriormente, elaborar un plan de actuación que permitiera mejorarla con criterios sostenibles. 
rismo, promovida por la Secretaría de Turismo y la Secretaría de Medio Ambiente y Recursos Naturales para fomentar la sustentabilidad de la actividad turística desde su implementación y la creación de un sistema de indicadores en municipios turísticos (Martínez y Ruiz, 2009). La ONU también registra experiencias en América del Norte, tanto en Estados Unidos como en Canadá, en el continente africano (Malawi, Ghana, Sudáfrica, Kenia, Nigeria, Senegal, Tanzania, Uganda, Zambia, Zimbabue, Mozambique, Marruecos o Túnez), en Asia (India, China, Japón, Corea del Sur, Filipinas, Mongolia, Malasia, Indonesia, Tailandia o Vietnam, entre otros) y en Oceanía (Australia y Nueva Zelanda).

\section{ALGUNOS LOGROS DE LA AGENDA 21}

En lo que a la participación ciudadana se refiere, en cierta medida y durante algunos años, la Agenda 21 (fundamentalmente la Agenda 21 Local) se convirtió en la gran referencia de las iniciativas político-sociales de promoción de la participación pública (Low et al., 2000), «hasta su fase de declive, que podríamos fijar en los comienzos de la década de 2010» (García-Montes y Arnanz, 2019: 115), en plena crisis económica internacional. Hasta entonces, la Agenda 21 era el proyecto «estrella» en cuanto a la participación de la ciudadanía a nivel institucional, facilitando canales de comunicación y expresión vecinal ante los problemas y propuestas de una localidad en relación al medio ambiente y el desarrollo sostenible. La ciudadanía de base y el tejido asociativo podían encontrar una herramienta donde adoptar un rol más cercano al de ciudadano activo que al de usuario de una Administración pública, superando una «concepción privatizadora del espacio [que] realmente está reservando a los ciudadanos el papel de clientes» (Boira Maiques, 2003: 322). Por tanto, sin participación en los asuntos públicos se pierde el estatus de ciudadanía y se sustituye por el de clientela. La Agenda 21, al menos en su planteamiento teórico, invitaba a los habitantes a aportar su visión subjetiva y vivida en la planificación sustentable de sus ciudades, y de esta manera se podría «fomentar un lazo identitario más fuerte entre población y espacio poblado» (García-Montes y Arnanz, 2019: 114), además de abrir un camino a la profundización de la democracia participativa.

Aquellas iniciativas de Agenda 21 con enfoques participativos más rigurosos han permitido que la ciudadanía de base (no asociada, no acostumbrada a participar en la actividad municipal, sin intereses políticos partidistas o sin más voluntad que contribuir a la mejora de su entorno, del medio ambiente y de la sustentabilidad local-global) pudiera ser partícipe de identificación de problemas y elaboración de propuestas para su 
hábitat residencial o laboral. Son experiencias de democracia participativa que han permitido ir más allá de un mero «maquillaje» orientado a cumplir superficialmente y como un trámite con la premisa de iniciar un diálogo con la ciudadanía, organizaciones y entidades, $\mathrm{y}$ han favorecido el desarrollo de verdaderos procesos de implicación ciudadana en lo público, tal y como establecía la Agenda 21 en sus postulados fundacionales. En términos cuantitativos, la participación, aunque escasa en comparación con el total de la población residente en una localidad, ha podido ser mayor que la habitual en la planificación de lo público (regularmente reservada a los niveles técnicos y políticos). Esto ya es un avance en sí mismo, a pesar de no lograr cifras de participación excesivamente altas (Martínez y Rosende, 2011; Verdugo-Perona y García Gómez, 2013), especialmente si se compara con otros procesos de intervención ciudadana, como la votación en unas elecciones o referéndums, o la asistencia a manifestaciones, acciones colectivas de protesta...

En cuanto al escenario para la participación que debía ofrecer la Agenda 21, más allá de los porcentajes de participación, lo más destacable es que ha contribuido a abrir una ventana a la incorporación de la opinión, demandas, expectativas y necesidades ciudadanas en la gestión municipal-institucional. Ventana que habitualmente no existía previamente. Y no se debe caer en el juego cuantitativo de la cantidad y la representatividad, propio de mediciones estadísticas, ya que los procesos de participación ciudadana no son equiparables a las encuestas. Lo que hay que reseñar es que el simple hecho de contar con más puntos de vista en el diseño y planificación de las políticas públicas ya constituye un elemento favorable. Especialmente porque son puntos de vista de una serie de actores sociales diferentes a los planificadores habituales, como suelen ser técnicos, políticos, expertos o representantes de colectivos formales.

\section{NIVELES DE PARTICIPACIÓN}

Cuando hablamos de participación, hablamos de tomar parte. Incluso, de sentirse parte (Pindado, 2009). Tomar parte o sentirse parte en los asuntos públicos comunes (comunitarios), tanto a nivel individual (personas físicas) como a nivel colectivo (asociaciones o entidades con cierto grado de organización). Para ello, se deben establecer una serie de mecanismos y canales de participación que permitan a la ciudadanía implicarse en lo procomún sin necesidad de pertenecer a las estructuras formales de gobierno ni «ostentar cargos o funciones públicas» (Díaz Aldret, 2017: 343). Si atendemos a la definición de participación de Hart (1993: 5), «la participación es la capacidad para expresar decisiones que sean reconocidas por el entorno social y que afec- 
tan a la vida propia y/o a la vida de la comunidad en la que uno vive», hay que decir que la participación en los procesos de Agenda 21 se ha quedado, en muchos de los casos, en un nivel de consulta (Garrido, 2005), sin dar el salto a la esfera de la toma de decisiones.

Tomando como referencia la escalera de la participación adaptada del modelo inicial de Arnstein (1969) para determinar los distintos niveles secuenciales hacia la participación, un primer peldaño lo constituye la información ${ }^{3}$, es decir, el derecho a ser informados. No obstante, esto no puede considerarse participación sensu stricto, más bien es un requisito previo, una condición que se tiene que dar para que pueda haber participación. La información favorece la toma de conciencia crítica, la creación de opinión y la transparencia, pero no puede quedarse en un mero instrumento legitimador o una operación estética (García-Montes, 2019: 18). El segundo peldaño es el de la consulta. Ya no se da una relación unidireccional entre Administración y administrados, como en el nivel de la información, sino que se permite un flujo bidireccional. Pero la consulta sobre la opinión ciudadana respecto a determinados asuntos públicos no implica compromiso o vinculación, y muchos procesos denominados participativos (como muchas Agendas 21) se limitan únicamente a consultar a los implicados y así dar un cierto barniz participativo que no traspasa el ámbito de las sugerencias para constituirse en realidades dentro de la gestión pública (García-Montes, ibid.). Un tercer peldaño es el de la toma de decisión, y aquí sí se puede estar hablando de participación real, efectiva y transformadora. Es decir, se permite a la ciudadanía compartir la toma de decisiones, ejercer el derecho a decidir sobre las cuestiones que le afectan. En este nivel de participación, el ciudadano, informado, consultado y escuchado, tiene la oportunidad de intervenir en un proceso de transformación social procediendo como sujeto activo y no como objeto o simple receptor pasivo utilizado de manera instrumental por los poderes institucionales (García-Montes, ibid).

Al final de la escalera, en un cuarto peldaño, se puede encontrar un nivel de participación no muy explorado todavía, pero donde se generan prácticas de democracia participativa o democracia directa, y donde se habilita a los ciudadanos la posibilidad de compartir responsabilidades (Lovan et al., 2017), una corresponsabilidad a través de un reparto del poder. Hablamos de la participación como cogestión, es

${ }^{3}$ En la propuesta planteada por Garrido y Martín (2006), también inspirada en Arnstein, se habla de un escalón previo en el que la participación se concibe como mera asistencia y consumo de programas, servicios, equipamientos, etc., que dan cuenta de los distintos derechos reconocidos a nivel público, generalmente en forma de necesidades (salud, transporte, zonas verdes, ocio...). En este nivel, el ciudadano es considerado como un cliente-usuario de servicios públicos. 
decir, la gestión compartida de algún asunto o actividad pública entre el sector institucional y la ciudadanía. O, incluso, autogestión (un nivel incluso superior, ya que en la cogestión el reparto de responsabilidades lo decide el actor principal, ejerciendo una mayor cota de poder), cuando la ciudadanía organizada se hace cargo y responsable de la gestión de alguna parcela de la vida pública. Este nivel de participación supone un proceso acumulativo de todos los peldaños anteriores de la escalera (información, consulta, toma de decisión), generando autoorganización (García-Montes, 2019: 19). O, como se apunta desde las actuales teorías y ciencias de la complejidad (Morín, 1990), desde la participación al máximo nivel se favorece un proceso de construcción de un sistema autorreflexivo que autoaprende de forma distinta a los sistemas que son programados o enseñados desde fuera. Según el análisis que se hace en este artículo, las Agendas 21 no alcanzaron con rigor, amplitud y garantías los dos últimos peldaños de esta escalera. Fueron procesos de consulta en los que, en ocasiones, se tuvo en cuenta algunas de las propuestas elaboradas participadamente por la comunidad local, pero no desde una óptica de coparticipar en la toma de decisiones sobre la planificación local ni cogestionar de manera (co)responsable lo público.

\section{¿POTENCIAL PLENAMENTE APROVECHADO?}

A pesar de la gran extensión de las Agendas 21 en todo el mundo y de sus contribuciones para ampliar y facilitar la participación ciudadana, si se pone el acento en la calidad de los procesos participativos, hay elementos que apuntan a considerar que muchas de las prácticas desarrolladas no han alcanzado verdaderas cotas de participación directa y de gobernanza participativa. La Agenda 21 ofrecía un potencial innovador y estructurado para aplicar criterios de sustentabilidad ambiental a las políticas públicas y a la planificación local contando con la participación ciudadana como eje central (McCully y McCoy, 1993). Una posibilidad para crear cultura democrática e implicativa en la población, para tejer sentido de pertenencia con el territorio, para promover hábitos, actitudes y conciencia proambiental desde lo local y, en definitiva, una posibilidad para hacer ciudades más sustentables y ecosistémicamente viables desde la gobernanza implicativa.

Pero debemos hablar de un potencial no aprovechado. Asumiendo el papel destacado que las Agendas 21 han jugado en la promoción de la participación ciudadana a nivel local (Carter y Darlow, 1997; Font, 2000; Selman, 2000; Pellizzoni, 2001), conviene poner de manifiesto que han tendido a ser casos excepcionales, y no la norma, en los que 
se ha logrado una verdadera implicación de amplios sectores de la población local (Gaye et al., 2001). Suele ser una afirmación bastante extendida la que señala que la participación ciudadana es uno de los principales déficits en los procesos de Agenda 21 (Arnanz et al., 2005).

La Agenda 21 no ha pasado, en muchas ocasiones, de promover una participación consultiva. Una participación que se ha podido utilizar por parte de los gobernantes para legitimar actuaciones (Jessop, 2003), dotar de cierto barniz democrático a la planificación local y cumplir con una moda de hacer partícipe a la población en diferentes asuntos municipales (moda y demanda al mismo tiempo). Una participación «concebida por la mayoría de gobernantes locales como consulta, apoyo o complemento para ejercer el gobierno; e incluso como mecanismo justificador de decisiones tomadas con anterioridad por políticos y técnicos» (Garrido, 2005: 64).

La falta de una verdadera voluntad política, unida al desconocimiento de un amplio sector de los gobernantes municipales respecto a qué es participación ciudadana, qué implica desarrollar un proceso participativo, qué condiciones se necesitan, qué recursos y capacidades hay que destinar y qué consecuencias y resultados hay que asumir, también han obstaculizado la promoción de procesos realmente participativos e implicativos que profundicen en un nivel decisional. Encontramos cuatro aspectos principales, habitualmente interrelacionadas e interdependientes, que conducen a una espiral de desafección institucional y política por parte de los gobernantes para impulsar procesos participativos:

- Falta de un verdadero interés, compromiso, voluntad y actitud política para propiciar verdaderos procesos participativos y de democracia directa o semidirecta.

- Desconocimiento, en algunos casos, por parte de los agentes encargados en primera y principal instancia de promover la participación ciudadana respecto a lo que realmente es participar en los asuntos públicos, sus dimensiones, sus implicaciones y su alcance.

- Temor por parte de los representantes gubernamentales respecto a ceder o a compartir una parte del ejercicio del poder y de la toma de decisiones con la ciudadanía.

- Falta de recursos económicos, técnicos y humanos para lograr altas cuotas de implicación y autoorganización ciudadanas.

Como se dice, esos elementos están interconectados: el desinterés genera desconocimiento y el desconocimiento genera desinterés, el desconocimiento genera temor y el 
temor genera desconocimiento y desinterés. Así mismo, la falta de voluntad e interés conlleva la escasa asignación de recursos... Y así continuaría el círculo vicioso.

\section{ALGUNOS INDICADORES PARA EVALUAR EL ALCANCE LOS PROCESOS PARTICIPATIVOS}

La Agenda 21, como herramienta con amplio potencial para fomentar procesos verdaderamente participativos, transformadores y autogestionados, no ha cumplido, en gran parte de los casos, las expectativas creadas y los planteamientos fundacionales con la que fue concebida. Para comprobar el alcance de un proceso participativo en el marco de las Agendas 21 se podrían aplicar una serie de indicadores que proponemos a continuación, los cuales permitirían testear si responde a los mínimos necesarios para considerar tal proceso como verdaderamente participativo:

- Grado de cobertura y accesibilidad. Es decir, el número y diversidad de actores sociales implicados y las facilidades ofertadas para participar (convocatorias y canales de participación abiertos). Una Agenda 21 con un alto grado de participación, no tanto en términos cuantitativos como cualitativos (acceso de diferentes posturas, puntos de vista e intereses), contribuiría a dar respuesta a la necesidad de ampliar la red de participantes más allá de las redes más activas e implicadas, que suelen ser las de los actores más vinculados a las cuestiones medioambientales (Blanco y Gomà, 2002). El enfoque diferencial debe recaer, más que en la cantidad (participación medida en términos cuantitativos), en la posibilidad que se debería abrir a cualquier ciudadano interesado para implicarse, opinar, proponer y participar en lo público. Es decir, se trata de que la participación esté a libre disposición del ciudadano como sujeto activo, corresponsable y accionista (Alguacil, 2005), y no sea un elemento meramente instrumental (Montecinos, 2017) para legitimar las actuaciones políticas desde una perspectiva de gobierno local gerencial-empresarial. Y las Agendas 21 que han fomentado una participación abierta, inclusiva y horizontal, generalmente han ofrecido tal posibilidad, frente a otros modelos de participación en Agendas 21 con un carácter más «elitista», «selectivo» (para asociaciones o grupos organizados, por ejemplo) o consultivo.

- Grado de participación (siguiendo la escalera de la participación, hasta qué nivel se llega). Es decir, si el proceso participativo permite superar el nivel de la mera consulta y se alcanza el nivel de la implicación en la toma de decisiones y la 
corresponsabilidad en la ejecución de acciones. Ello supone un compromiso y una voluntad política por apostar por la participación como recurso democrático para la autogestión y no como una consulta que sirva de apoyo a la labor de gobierno o como un elemento decorativo en la gestión pública que resulta electoralmente atractivo.

- Grado de logro de resultados. Es decir, el número de actuaciones decididas de manera participada que se ejecutan y su grado de importancia y trascendencia para la localidad. En muchas ocasiones, la participación no es vinculante y no genera resultados, y en otras tantas las prácticas se quedan en la propia realización de los procesos, sin resultados concretos que se materialicen en mejoras para la comunidad (Brugué, 2009).

- Grado de transparencia en la información, acceso a la misma por parte de la ciudadanía, difusión y grado de deliberación, apertura y ausencia de censura en la participación. Es necesario que la información circule, se comparta y se socialice (Montañés y Martín, 2017).

- Grado de continuidad del proceso y capacidad de permanencia frente a cambios externos (políticos o sucesos locales de otra índole). Es decir, que la participación no sea puntual (y meramente instrumental), sino que adquiera un estatus de transversalidad y permanencia en la gobernabilidad.

- Cómo son las normas que rigen el proceso participativo. Es decir, si son impuestas por un reglamento otorgado por la Administración; si existen unas normas pactadas con determinadas partes de la población; o si se establece un sistema autorreglamentado por grupos diversos de la población afectada (Observatorio de la Participación de Madrid, 2016).

- Grado de presencia de elementos de pedagogía social. Es decir, si ha habido formación específica (en participación, dinamización...); si la formación ha sido para todos los participantes o solo para el personal técnico (de la Administración o de las entidades sociales); si la formación es puntual o sistemática; o si ha habido especialistas y dinamizadores en el proceso (Observatorio de la Participación de Madrid, 2016).

Como se viene sosteniendo, la Agenda 21 ofrecía un amplio potencial para servir de plataforma de participación ciudadana. Otro asunto es si la ciudadanía ha podido, sabido o querido aprovecharla, cuestión que en algunos casos podrá ser imputable al desinterés del propio ciudadano (ICLEI, 2002), pero en otros podrá deberse a la falta de información, difusión, transparencia, mecanismos y canales para participar... facilitados desde las Administraciones. 


\section{¿PROYECTO ENTERRADO?}

El lector se habrá podido percatar de que en este apartado se habla de la Agenda 21 en tiempo pasado. Es algo intencional, ya que se considera que, por lo apreciado empíricamente, no se trata de un proyecto de presente y, previsiblemente, no lo sea tampoco de futuro. Al menos no con la denominación con que se ha conocido. Probablemente habrá iniciativas, acciones, proyectos y procesos encaminados a buscar una mayor sostenibilidad local implicando a la ciudadanía y a los diferentes agentes socioeconómicos de un territorio en procesos de gobernanza participativa. Pero mayormente ya no serán denominados Agenda 21, una iniciativa que tuvo su momento, disfrutó de su periodo de auge a mediados de la década de 2000 y que ha experimentado en los últimos años (desde mediados de la década de 2010) su fase de declive. Un declive que si bien no ha llegado a enterrar definitivamente el proyecto, dado que algunas experiencias siguen manteniendo viva la llama (aunque en un nivel de subsistencia tramitaria), sí ha rebajado de forma muy sobresaliente la atención mediática, el interés sociopolítico y la demanda ciudadana que suscita.

Es sintomático que en los Objetivos de Desarrollo Sostenible (ODS), establecidos por la Asamblea General de la ONU el 25 de septiembre de 2015, no se hace ni una sola mención a la Agenda 21, a pesar de que fue la propia ONU la que promovió tal proyecto veintitrés años antes. Tales objetivos se enmarcan en la Agenda 2030 para el Desarro1lo Sostenible («Transformar Nuestro Mundo: la Agenda 2030 para el Desarrollo Sostenible»), donde no hay ninguna referencia a la Agenda 21 en todo el documento, ni en la Declaración, ni en los Objetivos, ni en los Medios de implementación y la Alianza Global, ni en el Seguimiento y Revisión. A pesar de que en el punto 12 de la Declaración se señale: «We reaffirm all the principles of the Rio Declaration on Environment and Development» (ONU, 2015), la Agenda 21, tal y como se concibió en Río 92, no parece ser ya una herramienta válida para el futuro en una estrategia de desarrollo sostenible a nivel global con participación pública, ni siquiera para la propia organización que la creó.

\section{CONCLUSIONES}

Probablemente la Agenda 21 ya se ha ido, habiendo dejado sus luces: fue capaz de poner el medio ambiente y la sostenibilidad ecológica en la agenda pública y política internacional y local; se han implementado, bajo su auspicio, numerosas actuaciones de mejora de entornos urbanos y de conservación, recuperación y prevención de impactos 
ambientales; favoreció un acercamiento de parte de la población y de diferentes actores sociales y económicos a la sustentabilidad desde lo local; y permitió generar concienciación proambiental o, al menos, una mayor información tanto entre la ciudadanía como entre los sectores políticos y técnicos. Pero también ha dejado sus sombras, especialmente un potencial para abrir espacios de participación ciudadana no plenamente aprovechado.

Atendiendo, al menos, a sus postulados teóricos y la finalidad con la que nació, la Agenda 21 «presentaba un enorme potencial para convertirse en un escenario propicio para fomentar la participación ciudadana en el área medioambiental, conjugando dos dimensiones de creciente interés social y político: la sustentabilidad y la implicación de la población en los asuntos públicos» (García-Montes y Arnanz, 2019: 114). Con la llegada del nuevo siglo, y especialmente desde mediados de la década de 2010, han proliferado deseos, iniciativas, intereses, espacios, experiencias y discursos (al margen de la mayor o menor autenticidad, coherencia, credibilidad, rigor y compromiso de los mismos) reclamando una mayor implicación y participación de la ciudadanía en lo público, en algunos casos desde ópticas de democracia participativa que complemente a la democracia representativa. Y esto, que ya de por sí puede considerarse un logro y una nueva forma de afrontar las políticas públicas desde las Administraciones locales, ha tenido un motor de impulso en la Agenda 21. Y puede considerarse un paso más allá de una democracia meramente representativa y delegativa, sujeta a una participación electoral puntual y esporádica.

Se puede poner en la cuenta de logros de las Agendas 21 haber dado un paso importante hacia la gestión compartida de lo público (y si no tanto en la gestión, al menos en la planificación), aunque el paso haya sido más corto de lo que podría haber dado de sí el proyecto con base en su planteamiento inicial. Pero también es evidente que los mecanismos de participación ciudadana institucional (se excluyen los movimientos sociales, las iniciativas de acción colectiva de protesta y otros procesos de construcción democrática de la vida común de base, es decir, no promovidos desde la esfera institucional) existentes previos a la Agenda 21 no tuvieron tanto eco y extensión geográfica como la que tuvo la iniciativa surgida de la Cumbre de la Tierra de 1992.

\section{BIBLIOGRAFÍA}

Alguacil, J. (2005). «Los desafíos del nuevo poder local. La participación como estrategia relacional en el gobierno local». Polis Revista Latinoamericana, n. ${ }^{\circ} 12$, online, en: http://journals.openedition.org/polis/5614 
Arboit, M.E. y De Rosa, C. (2014). «Alcanzar la sostenibilidad ambiental, global y local del hábitat humano: el imperativo fundamental en el s. XXI». Espacio, Cultura y Sociedad, 16: 16, pp. 157-184, Universidad Nacional del Nordeste (Argentina).

Arnanz, L.; Belmonte, R.; García Montes, N. y Heras, P. (2005). «La Agenda 21: potencialidades y dificultades», en Garrido, F.J. (coord.): Desarrollo Sostenible y Agenda 21 Local. Prácticas, metodología y teoría. Madrid: IEPALA/CIMAS.

Arnstein, S.R. (1969). «A Ladder of Citizen Participation». Journal of the American Planning Association, . $^{\circ}$ 35: 4, pp. 216-224.

Bárcena, I. (2006). «¿Democracia participativa + desarrollo sostenible $=$ democracia ambiental? Preguntas y certidumbres para la democratización ecológica», en Encina, J. y Bárcena, I. (eds.): Democracia ecológica. Formas y experiencias de la participación en la crisis ambiental. Sevilla: UNILCO.

Blanco, I. y Gomà, R. (2002). «Proximidad y participación: marco conceptual y presentación de experiencias», en Blanco, I. y Gomá, R. (eds.): Gobiernos locales y redes participativas. Barcelona: Ariel.

Boira Maiques, J.V. (2003). «La participación ciudadana y el urbanismo ¿radicalizar la democracia o democratizar el espacio?», en Capel, H. (coord.): Ciudades, arquitectura y espacio urbano. Caja Rural Intermediterránea, n. ${ }^{\circ}$ 3, pp. 317-332.

Bosworth, T. (1993). «Local authorities and sustainable development». European Environment, . $^{\circ} 3$, pp. 13-17.

Brugué, Q. (2009). «Una administración que habla es una administración que piensa. De la gestión pública a la administración deliberativa», en Subirats, J. et al.: Participación ciudadana... para la administración deliberativa. Gobierno de Aragón.

Carter, N. y Darlow, A. (1997). «Local Agenda 21 and developers: are we better equipped to build a consensus in the 1990 's?». Planning Practice and Research, n. ${ }^{\circ} 12$ : 1, pp. 45-57.

Conama (2002). «Retos y dificultades en la implantación de las Agendas 21». Grupo de Trabajo n. ${ }^{\circ}$ 24, VI Congreso Nacional de Medio Ambiente, España.

Coria, L. (2007). «Herramientas de Planificación Ambiental Local: estado de situación y tendencias en la implementación de los Planes de Desarrollo Local Sustentable en Noroeste Argentino», online, en: www.sicbasa.com/rionda/eventosvirtuales/2007/6/ pon/lgc.doc.

Dahl, R. (1987). Un prefacio a la teoría democrática. México: Gernika

Díaz Aldret, A. (2017). «Participación ciudadana en la gestión y en las políticas públicas». Gestión y Política Pública, n. ${ }^{\circ}$ 26: 2, online, en: http://www.scielo.org.mx/ scielo.php?script=sci_arttext\&pid=S1405-10792017000200341 
Foladori, G. (2001). «Los problemas ambientales urbanos y sus causas». Revista Paranaense de Desenvolvimento, $\mathrm{n}^{\circ}$ 100, pp. 69-78.

Font, N. (2000). «Local y sostenible», en Font, N. y Subirats, J. (eds.): Local y sostenible. La Agenda 21 Local en España. Barcelona: Icaria.

García-Montes, N. (2019). «Abriendo caminos. Los procesos de participación ciudadana promovidos a nivel institucional en el ámbito local, como escuela de profundización democrática». Forum, Revista Departamento de Ciencia Política, n. ${ }^{\circ} 15, \mathrm{pp}$. 11-35, online en: https://revistas.unal.edu.co/index.php/forum/article/view/ $73368 / 70920$

García-Montes, N. y Arnanz, L. (2019). «Metodologías participativas para la planificación de la sostenibilidad ambiental local. El caso de la Agenda 21». Empiria, Revista de metodología de Ciencias Sociales, . $^{\circ}$ 44, pp. 109-133, online en: http://revistas.uned.es/index.php/empiria/article/view/25354/20335

Garrido, F.J. (2005). «Sostenibilidad y participación ciudadana en los procesos de Agenda 21 Local», en Garrido, F.J. (coord.): Desarrollo Sostenible y Agenda 21 Local. Prácticas, metodología y teoría. Madrid: IEPALA/CIMAS.

Gaye, M.; Diouf, L. y Keller, N. (2001). «Moving towards Local Agenda 21 in Rufisque». Environment \& Urbanization, n. ${ }^{\circ} 13: 2$, pp. 201-214.

Habermas, J. (1998). The Inclusion of the Other: Studies in Political Theory. Cambridge, Mass: MIT Press.

Hart, R.A. (1993). Children's participation: from tokenism to citizenship, Florence: UNICEF.

Hewitt, N. (1995). European Local Agenda 21 Planning Guide: How to engage in longterm environmental action planning towards sustainability, Freiburg: ICLEI.

ICLEI (2002). Segundo Informe de la Encuesta Agenda 21 Local. Secretaría para la Cumbre Mundial sobre Desarrollo Sustentable de la ONU.

Jessop, B. (2003). «Governance and Metagovernance: On Reflexivity, Requisite Variety and Requisite Irony», en Bang, H. (ed.): Governance, Governmentality and Democracy. Manchester: Manchester University Press.

Jones, M. y Stokes, T. (1998). «A comparision of the provision of Green spaces between urbanized áreas in two European localities, and the effects of the local Agenda 21 on their respective planning strategies». Managing Leisure, n. ${ }^{\circ}$ 3, pp. 71-84.

Lovan, W.R, Murray, M. y Shaffer, R. (2017). Participatory Governance. Planning, Conflict Mediation and Public Decision-Making in Civil Society, London: Routledge.

Low, N.; Gleeson, B.; Elander, I. y Lidskog, R. (2000). Consuming cities. The urban environment in the global economy after the Rio Declaration, London: Routledge. 
Martínez, M. y Rosende, S. (2011). «Participación ciudadana en las Agendas 21 locales: cuestiones críticas de la gobernanza urbana». Scripta Nova, Universidad de Barcelona, Vol. XV, n. ${ }^{\circ} 355$, online en: http://www.ub.edu/geocrit/sn/sn-355.htm.

Martínez, O. y Ruiz, J. (2009). «Las particularidades de la Agenda 21 para el turismo mexicano. Un análisis de la aplicación del sistema de indicadores de sustentabilidad en el municipio de Playas de Rosarito, Baja California». Gestión Turística, n. ${ }^{\circ}$ 12, Universidad Austral de Chile, pp. 9-29.

McCully, P. y McCoy, M. (1993). The Road from Río. An NGO Action Guide to Environment and Development, Utrecht: International Books/Wise.

Mebratu, D. (1998). «Sustainability and Sustainable Development: historical and conceptual review». Environmental Impact Assessment Review, 18(6), pp. 493-520.

Montañés, M. y Martín, P. (2017). «De la IAP a las Metodologías Sociopráxicas». Hábitat y Sociedad, n. ${ }^{\circ} 10$, pp. 35-52.

Montecinos, E. (2017). «Instituciones Políticas y Participación Social en el Espacio Local». Revista Austral de Ciencias Sociales, no 9, p. 3-14.

Morin, E. (1990). Introduction à la pensée complexe, París: ESF.

Observatorio de la Participación de Madrid (2016). «Jornada de evaluación de la participación ciudadana. Resumen de Actas y Propuestas de futuro», online en: https:// observatorioparticipacionmadrid.com/

ONU (1992). Programa 21. Nueva York: Naciones Unidas, Departamento de Asuntos Económicos y Sociales, División de Desarrollo Sostenible.

- (2015). Transformar nuestro mundo: la Agenda 212030 para el Desarrollo Sostenible, Nueva York: Asamblea General de Naciones Unidas.

O'Riordan, T. y Voisey, H. (1998). The Transition to Sustentability: the politics of Agenda 21 in Europe. London: Earthscan Publications.

Pellizzoni, L. (2001). «The myth of the best argument: power, deliberation and reason». British Journal of Sociology, $\mathrm{n}^{\circ}$ 52: 1, pp. 59-86.

Pindado, F. (2009). «La participación ciudadana, la vida de las ciudades», en Subirats, J. et al.: Participación ciudadana... para la administración deliberativa. Gobierno de Aragón.

Robles de Benito, R.; Carabias Lillo, J. y Arellano-Guillermo, A. (2008). «Agenda 21 y descentralización en México», en Fraga, J. et al. (eds.): Descentralización y manejo ambiental. Gobernanza costera en México. México DF: Plaza y Valdés.

Schumpeter, J.A. (1983 [1942]). Capitalismo, socialismo y democracia. Barcelona: Orbis.

Selman, P. (1998). «Local Agenda 21: substance or spin?». Journal of Environmental Planning and Management, $\mathrm{n}^{\circ}$ 41: 5, pp. 533-54. 
Twyman, C. (1998). «Rethinking community resource management: managing resources or managing people in western Bostwana?». Third World Quarterly, n. ${ }^{\circ}$ 19: 4, pp. 745-770.

Tyler Miller, G. (2007). Ciencia ambiental: desarrollo sostenible. Un enfoque integral, México: Thomson.

Verdaguer, C. (2000). «De la sostenibilidad a los ecobarrios». Boletín $C F+S$, n. $^{\circ} 14$, online en: http://habitat.aq.upm.es/boletin/n14/acver.html

Verdugo-Perona, J. y García Gómez, J. (2013). «Participación Ciudadana en la Agenda 21 Local. Estudio de caso de Sagunto (Valencia)». Uni-Pluri/Versidad, n. ${ }^{\circ} 13$, pp. 102-113. 